\title{
Solving the Phase Problem in X-Ray Near-Field Holography Beyond the Assumption of Weak Objects
}

\author{
$\underline{\text { Johannes Hagemann }}{ }^{1, *}$, Mareike Töpperwien ${ }^{2}$ and Tim Salditt ${ }^{2}$ \\ 1. X-ray Nanoscience and X-Ray Optics, DESY, Notkestraße 85, 22607 Hamburg \\ 2. Institut für Röndgenphysik. Universität Göttingen, Friedrich-Hund-Platz 1, 37077 Göttingen \\ * johannes.hagemann@desy.de
}

X-ray near-field holography (NFH) is a coherent propagation-based phase-contrast microscopy technique for imaging a wide variety of samples. It is based on Gabor's idea for holography [1]. NFH offers the combination of high penetration depth, high resolution and tunable field of view [2]. It can be implemented at laboratory [3] and synchrotron [4] setups.

Recovering the actual image of the specimen involves solving an ill-posed inverse problem. It emerges from the loss of the phases of the complex valued wave field during the intensity-only measurement process. Simple reconstruction techniques as the plain back propagation of the measurement to the plane of the object, i.e. the so-called holographic reconstruction, are spoiled by artifacts as the twin image. This fostered the need for advanced reconstruction techniques to obtain artifact-free and quantitative images.

For NFH imaging experiments at synchrotrons using hard x-rays, phase retrieval is largely based on the single step reconstruction utilizing the contrast transfer function (CTF) approach, as introduced almost twenty years ago [5]. Notwithstanding its tremendous merits, this scheme makes stringent assumptions on the optical properties of the object, requiring in particular a weakly varying phase.

Figure 1 shows a comparison of the power spectral densities (PSD) for spheres of different diameter. The PSDs have been computed from data obtained at the Göttingen instrument for nano imaging with X-rays (GINIX) [6] located at the beamline P10 of PETRA III. The quasi point source illumination has been generated by a two-dimensional waveguide of $40 \mathrm{~nm}$ cross section. The photon energy was set to $8 \mathrm{keV}$, thus the maximum phase shift for polysterene is $0.47,0.79,1.01,2.36 \mathrm{rad}$ for the $3,5,7,15 \mu \mathrm{m}$ spheres, respectively. For increasing size of the spheres, associated with an increase of the maximum phase shift, the minima and maxima shift their position from the prediction of the CTF. This mismatch leads to a significant loss in image quality, as the assumptions for CTF phase retrieval are violated for large spheres. Figure 2 (a) depicts the CTF reconstruction of the $15 \mu \mathrm{m}$ spheres. The necessary data diversity for the reconstruction has been achieved by using a four propagation distances data set at Fresnel numbers $F=\Delta x^{2} /(\lambda z)=\{1.59,1.56,1.49,1.33\} \cdot 10^{-3}$, with respect to the effective size of one pixel $\Delta \mathrm{x}$ in the object plane.

Using an iterative phase reconstruction algorithm, as they are routinely applied in far-field coherent imaging, we can obtain a highly improved image quality. The iterative scheme was in this case the simple method of alternating projections (AP) [7] and used the same input for reconstruction as CTF. Figure 2 (b) shows the reconstruction obtained by AP. The constraint on the measurements has been computed in the standard way, i.e. propagating forward, adapting to the measured amplitudes while the phases are kept and propagating backwards. The object constraint has been switched after 1000 iterations from a constraint on the amplitude $A \leq 1$, leaving the phases unchanged, to a range constraint 
on the phase $\phi \leq 0$ and $A \leq 1$ for another 1000 iterations. The reconstruction finished with another 1000 iterations of the first constraint.

The AP reconstruction shows nearly homogenous spheres in contrast to the CTF reconstruction. The drawback of the iterative approach is the high number of iterations, which are especially required to reconstruct the low spatial frequencies. With the advent of new computational hardware, especially general purpose graphical processing units, the computation time of iterative algorithms can be drastically reduced. In conclusion the routinely application of iterative phase retrieval to even large dataset as from tomography becomes feasible with new hardware and can provide high quality reconstructions.

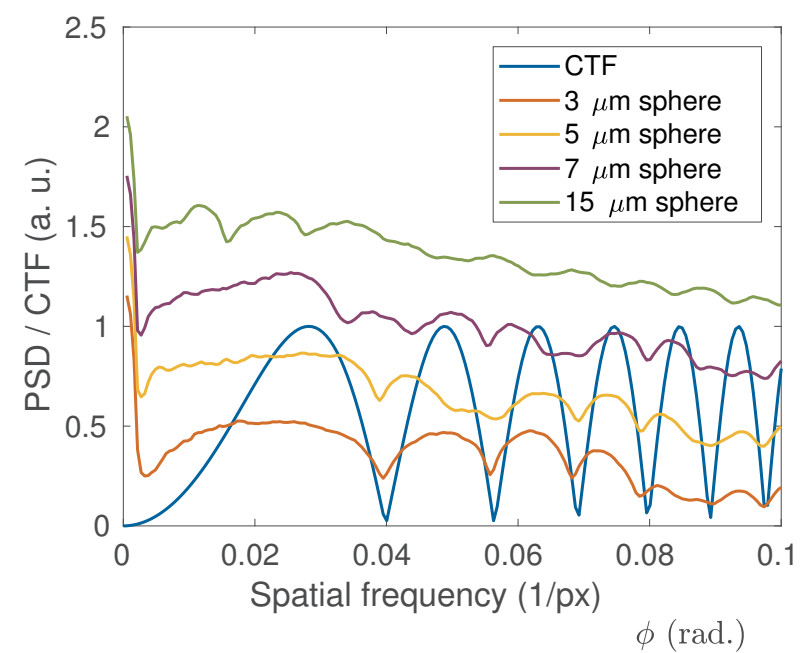

Figure 1. Comparison of the PSD for spheres of different diameters at Fresnel number $\mathrm{F}=1.59 \cdot 10^{-3}$ with the analytically computed CTF. The PSD curves have been normalized and shifted for clarity.
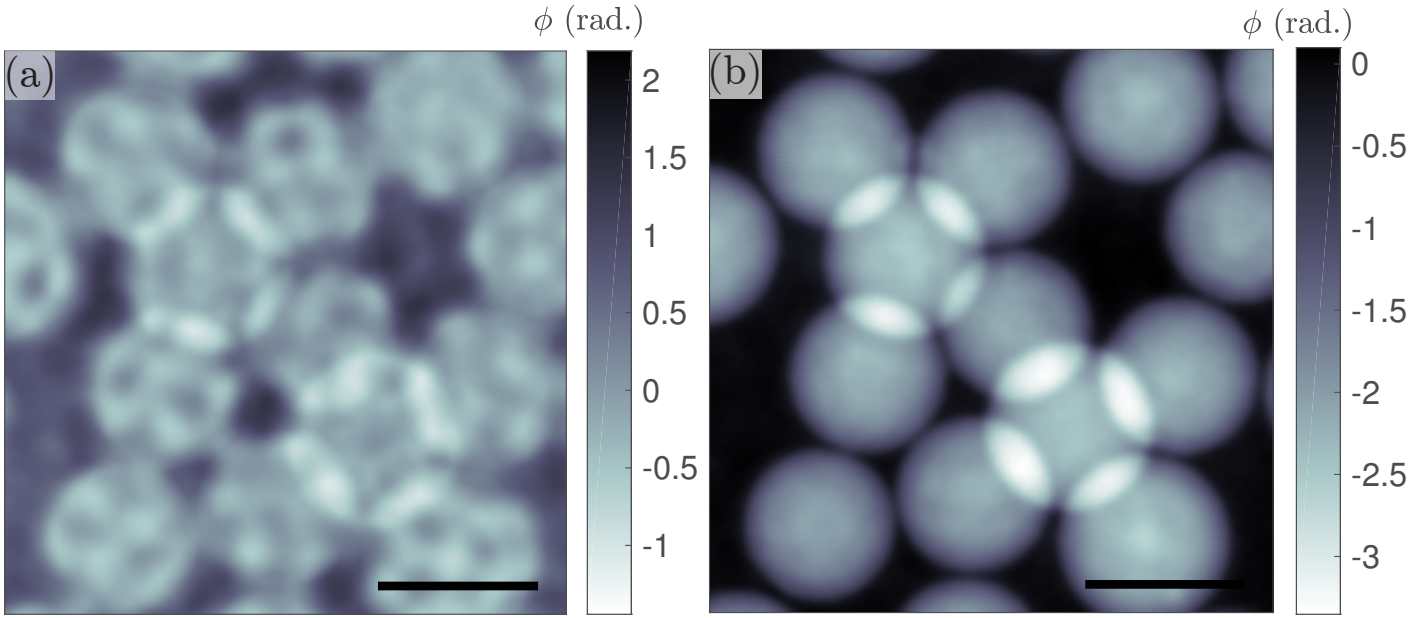

Figure 2. Comparison of the reconstructions for the $15 \mu \mathrm{m}$ spheres. (a) Phases obtained by CTF. (b) Iteratively obtained reconstruction. The scale bar in (a) and (b) indicates $15 \mu \mathrm{m}$.

[1] D. Gabor, Nature 161 (1948), 777

[2] M. Krenkel et al., Sci. Rep. 5 (2015), 09973

[3] M. Bartels et al., Appl. Phys. Lett. 103 (2013), 083703

[4] A. Snigirev et al., Rev. Sci. Instrum. 66 (1995), 5486 - 5492

[5] Cloetens et al., Appl. Phys. Lett. 75 (1999), 2912-2914

[6] T. Salditt et al., J. Synchrotron Radiat. 22 (2015), 867 - 878

[7] J. V. Neumann, Ann. Math. 50 (1949), 401 - 485 